\title{
Local Binary Pattern Guided Bilateral Spatial Error Reconstruction
}

\author{
Chen Yao, Lijuan Hong, Yunfei Cheng and Jin Huang \\ The Third Research Institute of Ministry of Public Security \\ Shanghai, China \\ e-mail: yaochensing@126.com
}

\begin{abstract}
In many communication occasions, packet loss or data corruption often occurs. Data incompleteness results in video degradation, which greatly affect visual quality. Error concealment is needed for the robustness of video transmission. In this paper, we introduce a local binary pattern guided bilateral spatial error concealment. In the computation of local search window, neighboring local binary patterns are computed. The computed binary value is forwarded to bilateral distance weight framework. In bilateral weight computation framework, geometric distance of neighboring patches is replaced by Hamming distance of neighboring local binary patterns. Finally, favorable results of the proposed algorithm are shown in experiments comparing with several competitors.
\end{abstract}

Keywords-Packet loss; Video degradation; Error concealment;Bilateral filter; Local binary pattern

\section{INTRODUCTION}

Transmission of video streams over error prone channels is not robust enough due to packet loss and error propagation. Error concealment is a process of restoring lost data by employing data redundancy. Linear interpolation scheme is often used as an effective method for smoothing image. Furthermore, some adaptive interpolations are presented to achieve maximum smoothness [1], [2]. As a rule, these methods reconstruct lost data from its neighbors in an interpolation surface. But, recovered data often contains high frequency components, which result in blurry in image. [3] and [4] proposed a fuzzy logic reasoning approach, which recovers the low frequency information with neighboring blocks. And high frequency information is interpreted on complicated textures and edges. In fuzzy logic reasoning, local search window iteration is performed for optimal output. Projection onto convex sets(POCS) is proposed by using block classifier to determine edge directions instead of imposing a smoothness constraint [5]. POCS can get satisfactory results when the lost data is characterized by a single dominant edge direction. In [6], a multi-scale approach is presented with a coarse-to-fine replenishment scheme. However, iterative computation is very time consuming and is hard for real-time application. In [7], geometric structure of missing data is inferred from neighboring data based on directional interpolation. The missing data is recovered by directional interpolation for keeping edge consistency. Heuristically, edge pixels selection is based on angle information. Except all the spatial error concealment mentioned above, more spatial error concealments is introduced in [2], [8], [9], [10], [11], [12], [13] etc. For temporal error concealment, residual DCT coefficients and motion vector are often lost due to transmission error. Many approaches are proposed for recover lost motion vectors. In [14], a side matching criterion is presented for motion vector selection based on minimization of matching distortion. Block matching scheme is used to estimate lost motion vector. Furthermore, advanced motion compensation is applied for motion vector recovery. In addition to temporal error concealment, spatiotemporal based methods are shown in [15]. Unlike previous methods, an adaptive interpolation method is used in [16] with a sequential recovery scheme.

In this paper, inspired by [15], [16] and [17], we present a local binary pattern guided bilateral spatial error concealment. The image data in the corrupted region are computed with a weighted average strategy in a local window. In bilateral filtering framework, geometric weight and radiometric weight are main computation overhead for bilateral algorithm. In our proposed algorithm, geometric weight is replaced by a hamming distance computation guided by local binary pattern. Obviously, we adopt a transformed hamming distance as a metric for different patches. The improved geometric weight is better than previous Euclidean distance because the feature of description of local image structure with local binary pattern. As a consequence, we have better recovery of the missing data at the cost of increased complexity from computing the local binary patterns.

The remainder of this paper is organized as follows. Description of algorithm is presented in the second section. The experimental results are shown in the third section and finally, the final section concludes the paper.

\section{The PROPOSED ALGORITHM}

In this section, firstly, a processing framework of bilateral filter is introduced. Then, local binary pattern related theory is reviewed. After that, an improved geometric weight is introduced in bilateral filter. Finally, an adaptive weight scheme is used to speed bilateral weight computation.

\section{A. Bilateral Filter Formulation}

Mathematically, a bilateral filter is denoted as

$$
B F[I]_{p}=\frac{1}{W_{p}}=\sum_{q \in S} G_{\sigma_{s}}(\|p-q\|) G_{\sigma_{r}}\left(\left|I_{p}-I_{q}\right|\right) I_{q} \text {, }
$$


where, $B F[\cdot]$ is denoted as bilateral operator. $W_{p}$ is normalization factor, which ensures pixel weights sum to 1.0. $W_{p}$ is defined as follows

$$
W_{p}=\sum_{q \in S} G_{\sigma_{s}}(\|p-q\|) G_{\sigma_{r}}\left(\left|I_{p}-I_{q}\right|\right) .
$$

where, parameters $\sigma_{s}$ and $\sigma_{r}$ specify the amount of filtering for the input image. $G_{\sigma_{s}}$ is spatial Gaussian weighting that decrease the influence of distant pixels. $G_{\sigma_{r}}$ is a rang Gaussian, which measures the radiometric distance between $I_{p}$ and $I_{q} \cdot G_{\sigma_{s}}(\|p-q\|)$ is geometric weight of filter. And $G_{\sigma_{r}}\left(\left|I_{p}-I_{q}\right|\right)$ is radiometric weight of filter.

\section{B. Local Binary Pattern Guided Geometric Weight Computation}

After a brief introduction of classical bilateral filter above, local binary pattern guided weight computation is detailed in this section. In fact, image self-similarity is used by image spatial domain formulation. The missing data can be recovered by similar pixels in spatial domain. In the following part, we first introduce the definition of local binary pattern (LBP) and then propose LBP guided bilateral filter using transformed geometric weight, which can regarded as a combination of bilateral filter and LBP.

We use the LBP [17] as texture descriptor of image processing pixels in local slide window. Essentially, the histogram of binary patterns computed over an image region is utilized in LBP. The LBP operator redefines each pixel by the relative grey levels to its neighboring region. The LBP value is set to one when the gray level of the neighboring pixel is higher or equal than current pixel. Otherwise, it is set to be zero. LBP describes the result over neighborhood as a binary pattern, mathematically defined by:

$$
L B P_{R, N(x, y)}=\sum_{i=0}^{N-1} s\left(n_{i}-n_{c}\right) 2^{i}, s(x)=\left\{\begin{array}{l}
1, \quad x \geq 0 \\
0, \text { otherwise }
\end{array} .\right.
$$

Where, $n_{c}$ is the gray level of the center pixel of a local neighborhood and $n_{i}$ corresponds to the gray levels of $N$ equally spaced pixels on a circle of radius $R$. In Fig.1, three circular neighbors are listed. In our paper, we adopt the first binary pattern (i.e. $P=8, R=1$ ). Eq.3 means that the signs of the differences in a neighborhood are interpreted as $N$-bit binary number, resulting in $2^{N}$ distinct values for the binary pattern. We then define the Hamming distance $D_{\text {hamming }}(p, q)$ from current point to neighboring point as

$$
D_{\text {hamming }}(p, q)=\operatorname{Hamming}\left(L B P_{R, N}(p), L B P_{R, N}(q)\right) \text {. }
$$

$p, q$ are the pixel indexes in local search window, respectively. From the definition of LBP hamming distance, we infer a new bilateral filtering framework, in which geometric weight is updated by the hamming distance. The updated bilateral filter is defined as follows,

$$
B F[I]_{p}=\frac{1}{W_{p}}=\sum_{q \in S} G_{\sigma_{s}}\left(D_{\text {hamming }}(p, q)\right) G_{\sigma_{r}}\left(\left|I_{p}-I_{q}\right|\right) I_{q} \text {. }
$$

where, original Euclidean distance is replaced with a LBP guided hamming distance.

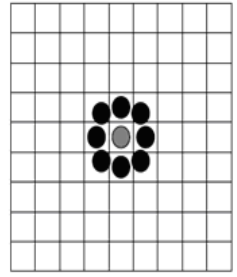

$P=8, R=1.0$

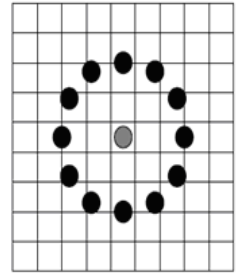

$P=12, R=2.5$

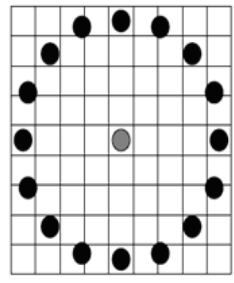

$P=16, R=4.0$
Fig. 1. Three circular neighborhoods for different pattern.

\section{Radiometric Weight Switch Strategy}

In addition to the improvement of geometric weight, we also give a radiometric weight switch strategy. We use pixel gradient to represent the similarity between two neighboring pixels. And the gradient $\nabla v$ is used as a switch toggling weight for radiometric weight computation, which is defined as

$$
\nabla v=\sqrt{\nabla v_{x} \times \nabla v_{x}+\nabla v_{y} \times \nabla v_{y}},
$$

where, $\nabla v_{x}$ and $\nabla v_{y}$ are horizontal and vertical gradient respectively. The key idea of this scheme is that radiometric weight does not need to be compute when $\nabla v$ is less than a certain threshold. The computation strategy of radiometric weight is described as the following,

$$
\text { Radiometric }=\left\{\begin{array}{l}
G_{\sigma_{r}}\left(\left|I_{p}-I_{q}\right|\right), \overline{\nabla v}>T \\
0, \quad \text { otherwise }
\end{array}\right.
$$

In our implementation, threshold $T$ is equal to 6 .

\section{EXPERIMENTS}

In this section, the benefits of error concealment results of the proposed algorithm are shown. Meanwhile, we provide our comparisons with some competitors under an objective evaluation. Fig. 2 contains different error pattern for error concealment test. Fig.2(a) is a error pattern of 50\% isolated block loss. Fig.2(c) is a error pattern of $30 \%$ isolated block loss. Fig.2(b) is a error pattern of $50 \%$ random mixed block loss. Fig.2(d) is a error pattern of $10 \%$ random mixed block loss. Fig. 3 is the error concealment results based on the error pattern in Fig.2. Subjectively, our method gets a good visual effect from test pattern. Objective evaluation results are also shown in Table I. We use PSNR as image quality 
assessment. And, the PSNR computation is only applied on the restored blocks. From the experimental results, the degraded blocks can be well reconstructed. As compared to previous error concealment algorithm [18] [13] [5] and [15], our proposed algorithm obtains highest PSNR. Parameters adjustment is not needed in our algorithm, which can be employed for different block loss rate, different block size and other conditions.

TABLE I. PERFORMANCE COMPARISON

\begin{tabular}{|c|c|c|c|c|c|}
\hline $\begin{array}{c}\text { Error } \\
\text { pattern }\end{array}$ & method[18] & method[13] & method[5] & method[15] & ours \\
\hline Fig2.(a) & 23.11 & 24.13 & 23.34 & 24.52 & $\mathbf{2 5 . 5 1}$ \\
\hline Fig2.(b) & 19.47 & 20.56 & 20.14 & 20.63 & $\mathbf{2 1 . 7 4}$ \\
\hline Fig2.(c) & 18.77 & 19.60 & 20.08 & 20.54 & $\mathbf{2 1 . 9 9}$ \\
\hline Fig2.(d) & 22.82 & 23.90 & 23.62 & 24.44 & $\mathbf{2 5 . 3 7}$ \\
\hline
\end{tabular}

\section{CONCLUSION}

In this paper, we present a novel error concealment algorithm built on local binary pattern guided bilateral filter framework. We build a new weight computation approach through introducing hamming distance as weight metric. Radiometric weight switching scheme is also applied in our method in order to simplify the whole computation. Experiments demonstrate the effectiveness and efficiency of our error concealment algorithm.

\section{ACKNOWLEDGMENT}

This work was supported by Science and Technology Innovation Foundation of Science and Technology Commission of Shanghai Municipality (12DZ0503300). Our work also was partly supported by Technical Standards Special Foundation of Science and Technology Commission of Shanghai Municipality (11dz0503600)

\section{REFERENCES}

[1] Y. Wang, Q.F. Zhu, and L. Shaw, "Maximally smooth image recovery in transform coding," Communications, IEEE Transactions on, vol. 41, no. 10, pp. 1544-1551, 1993.

[2] Y. Wang and Q.F. Zhu, "Signal loss recovery in dct-based image and Video codecs," in Visual Communications,'91, Boston, MA. International Society for Optics and Photonics, 1991, pp. 667-678.

[3] X. Lee, Y.Q. Zhang, and A. Leon-Garcia, "Image and video reconstruction, using fuzzy logic," in Global Telecommunications Conference,1993, including a Communications Theory MiniConference. Technical Program Conference Record, IEEE in
Houston. GLOBECOM’93., IEEE. IEEE, 1993, pp. 975-979.

[4] X. Lee, Y.Q. Zhang, and A. Leon-Garcia, "Information loss recovery for block-based image coding techniques-a fuzzy logic approach," Image Processing, IEEE Transactions on, vol. 4, no. 3, pp. 259-273, 1995.

[5] H. Sun and W. Kwok, "Concealment of damaged block transform coded images using projections onto convex sets," Image Processing, IEEE Transactions on, vol. 4, no. 4, pp. 470-477, 1995.

[6] S. Belfiore, L. Crisa, M. Grangetto, E. Magli, and G. Olmo, "Robust and edge-preserving video error concealment by coarse-to-fine block replenishment," in IEEE INTERNATIONAL CONFERENCE ON ACOUSTICS SPEECH AND SIGNAL PROCESSING. IEEE; 1999, 2002, vol. 4, pp. 3281-3284.

[7] W. Zeng and B. Liu, "Geometric-structure-based error concealment with novel applications in block-based low-bit-rate coding," Circuits and Systems for Video Technology, IEEE Transactions on, vol. 9, no. 4, pp. 648-665, 1999.

[8] J.W. Park, J.W. Kim, and S.U. Lee, "Dct coefficients recovery-based error concealment technique and its application to the mpeg-2 bit stream error," Circuits and Systems for Video Technology, IEEE Transactions on, vol. 7, no. 6, pp. 845-854, 1997.

[9] J.W. Park and S.U. Lee, "Recovery of corrupted image data based on the nurbs interpolation," Circuits and Systems for Video Technology, IEEE Transactions on, vol. 9, no. 7, pp. 1003-1008, 1999.

[10] A. Raman and M. Babu, "A low complexity error concealment scheme for mpeg-4 coded video sequences," in IEEE Symposium on Multimedia Communications and Signal Processing. Citeseer, 2001.

[11] A. Katsaggelos and N. Galatsanos, Signal recovery techniques for image and video compression and transmission, Springer, 1998.

[12] X. Li and M.T. Orchard, "Novel sequential error-concealment techniques using orientation adaptive interpolation," Circuits and Systems for Video Technology, IEEE Transactions on, vol. 12, no. 10, pp. 857-864, 2002.

[13] D. Agrafiotis, D.R. Bull, and C.N. Canagarajah, "Enhanced error concealment with mode selection," Circuits and Systems for Video Technology, IEEE Transactions on, vol. 16, no. 8, pp. 960-973, 2006.

[14] W.M. Lam, A.R. Reibman, and B. Liu, "Recovery of lost or erroneously received motion vectors," in Acoustics, Speech, and Signal Processing, 1993. ICASSP-93., 1993 IEEE International Conference on. IEEE, 1993, vol. 5, pp. 417-420.

[15] G. Zhai, J. Cai, W. Lin, X. Yang, and W. Zhang, "Image error concealment via block-based bilateral filtering," in Multimedia and Expo, 2008 IEEE International Conference on. IEEE, 2008, pp. 621624

[16] M. Elad, "On the origin of the bilateral filter and ways to improve it," Image Processing, IEEE Transactions on, vol. 11, no. 10, pp. 11411151, 2002.

[17] T. Ahonen, J. Matas, C. He, and M. Pietik"ainen, "Rotation invariant image description with local binary pattern histogram fourier features,” Image Analysis, pp. 61-70, 2009.

[18] Z. Wang, Y. Yu, and D. Zhang, "Best neighborhood matching: an information loss restoration technique for block-based image coding systems,” Image Processing, IEEE Transactions on, vol. 7, no. 7, pp.1056-1061, 1998. 


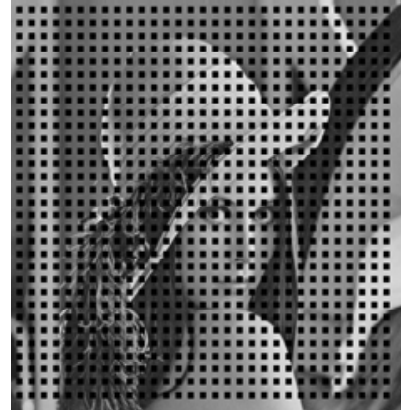

(a)

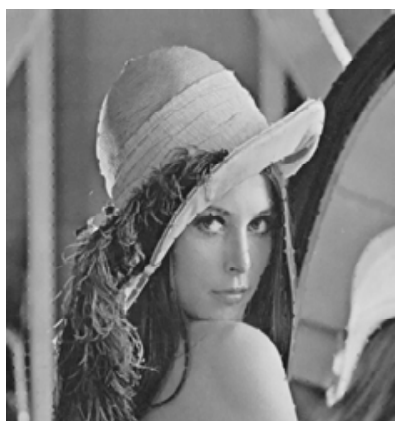

(a)

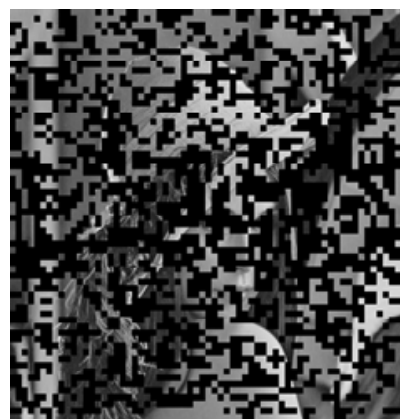

(b)

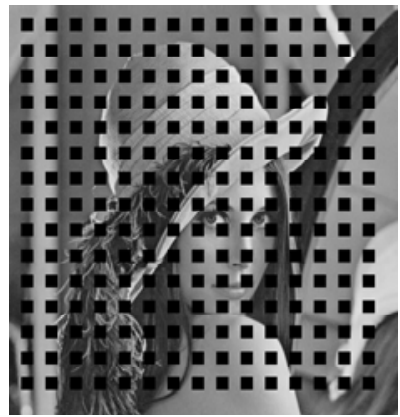

(c)

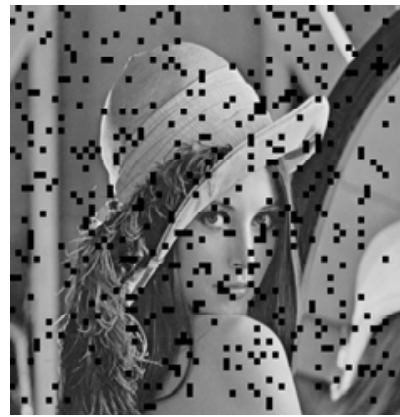

(d)

Fig. 2. error patterns for error concealment.

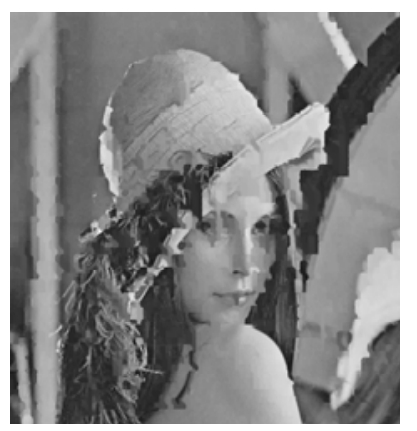

(b)

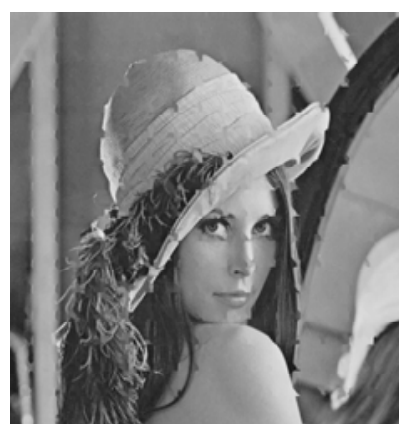

(c)

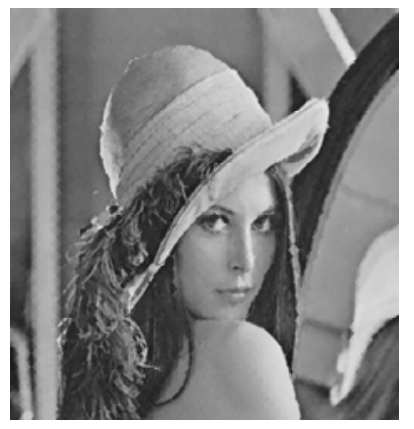

(d)

Fig. 3. error concealment results for error patterns in Fig. 2. 